\title{
The gradient structure of a flow: I
}

\author{
C. CONLEY† \\ Mathematical Sciences Department, IBM Thomas J. Watson Research Center, \\ Yorktown Heights, New York, USA
}

Abstract. Each flow dominates a unique gradient flow (the gradient part) which in turn dominates any other gradient flow dominated by the original flow. The set of points carried to rest points of the gradient part (the chain recurrent set) is characterized in terms of a recurrence relation. Filtrations of the given flow correspond to those of the gradient part.

\section{Introduction}

The basic object of study here is the collecticn of flows on a compact metric space which will be called the state space. This collection of flows is itself a complete metric space when endowed with the compact-open topology. From a general point of view the aim is to study mathematical entities which are constructed in terms of the state space and the space of flows, and in particular ones which pay attention to the topologies.

In the background it is assumed that the flow provides an imperfect model of some other object being studied (for example, an electrical network) and that the entities constructed relate to properties of this object (as, for example, the stable equilibria correspond to observed states of the network). However, such a point of view will not be allowed to hamper the development here.

An example of such an entity is the function which assigns to each flow its collection of closed invariant sets. This pays attention to the topology in that it an upper semicontinuous function on the space of flows [5]. Another function, defined on the product of the state space and the space of flows, assigns to each pair the $\omega$-limit set of the state with respect to the flow. This function does not pay attention to the topology in an easily describable way; however, a possible replacement is described below which is upper semicontinuous in both variables (\$ 3.4).

A somewhat different example is provided by the filtrations of the flow. A filtration can be defined to be a finite decreasing sequence of closed subsets of the state space, each of which is carried properly interior to itself by the flow. In a natural way, a sheaf over the space of flows can be constructed whose stalks consist of algebraic structures (graphs, cohomology algebras and homomorphisms) defined in terms of the filtrations. The dependence on the topologies is manifest in the sheaf structure (see [3], [4], [6] and [7]).

It is this last entity, the sheaf of gradient structures on the space of flows, which is of primary interest here. The first step is to relate the existence of filtrations to

+ The author was on leave from the University of Wisconsin at the time this paper was written. 
the recurrence properties of orbits, and this is carried out in part I. In part II the sheaf is constructed and some problems are discussed in which the algebraic aspects play a role.

These problems generally concern a one- or two-parameter family of flows for which it is of interest to distinguish parameter values where the stalk of the sheaf changes in some way. Such a change corresponds in these problems to the existence of an orbit of special interest such as a homoclinic or heteroclinic orbit. In turn, this orbit corresponds to a progressive wave solution of a partial differential equation.

This first part is divided into five sections, in the first of which is listed the notation. The second section contains a treatment of attractors designed to fit the remainder of the work. In the third section the relevant notion of recurrence is introduced. The main points are the definition of a generalized $\omega$-limit and recurrent set in terms of $(\varepsilon, t, f)$-chains, and the characterization of the former as the smallest quasi-attractor containing the usual $\omega$-limit set. In the fourth section the 'gradient part' of a flow is described. Flows could be classified in terms of their gradient parts; some of the significance of such a classification is seen in the statement that all recurrent flows fall into the same (the trivial) class, and all flows which are ' $\omega$-similar' (4.3A) under the domination relation have similar gradient parts. This implies in particular that the algebraic structures associated to the filtrations are the same (thus invariants of the similarity class), as will be shown in the second part. In the final section Morse decompositions are defined and related to the existence of filtrations.

\section{Notation}

1.1. $X$ denotes a compact metric space with metric $\rho$. The metric on $X \times X$ is denoted by $\rho$ also.

For $Y \subset X, \mathrm{Cl} Y, \partial Y$, Comp $Y$, Int $Y$ and Ext $Y$ denote respectively the closure, boundary, complement, interior and the interior of the complement of $Y$.

If $A, B \subset X, A \backslash B \equiv A \cap$ Comp $B$.

1.2. Flows on $X$ are denoted by $f=f: X \times R \rightarrow X$. For $Y \subset X$ and $J \subset R, Y \cdot J \equiv$ $f(Y \times J), f$ being known from the context.

To each flow $f$ there corresponds the backward flow $f^{*}$ defined by $f^{*}(x, t)=$ $f(x,-t)$. Note $f^{* *}=f$.

1.3. A set $Y$ is invariant under $f$ if $Y \cdot R=Y$. If $Y$ is invariant, then $\mathrm{Cl} Y, \partial Y$, Comp $Y$, Int $Y$ and Ext $Y$ are also, and all are invariant under $f^{*}$ as well. Also $f \mid Y$ will denote the restriction $f \mid Y \times R \rightarrow Y$.

A rest point of $f$ is a point which is an invariant set. $C(f)$ denotes the set of rest points ( $C$ stands for 'critical'; in the usual gradient case critical points correspond to rest points).

1.4. For $Y \subset X, \omega(Y, f) \equiv \bigcap_{t \in R} \mathrm{Cl}\left\{Y \cdot\left(t^{\infty}\right)\right\}$. Then $\omega(Y, f)$ is a closed invariant set and is connected if $Y$ is. For $Y \equiv\{x\}, \omega(x, f)$ is the usual $\omega$-limit set of $x$. The corresponding $\alpha$-limit set will always be denoted by $\omega\left(x, f^{*}\right)$.

1.5. The set $\{y \mid \omega(y, f) \cap Y \neq \varnothing\}$ could then be denoted $\alpha(Y, f)$. However, to avoid 
confusion, $\tilde{\alpha}(Y, f)$ will be used. This set is obviously invariant, but is not necessarily closed even when $Y$ is.

Observe that generally $\tilde{\alpha}\left(Y_{1} \cap Y_{2}, f\right) \subset \tilde{\alpha}\left(Y_{1}, f\right) \cap \tilde{\alpha}\left(Y_{2} f\right)$ and $\tilde{\alpha}\left(Y_{1} \cup Y_{2}, f\right)=$ $\tilde{\alpha}\left(Y_{1}, f\right) \cup \tilde{\alpha}\left(Y_{2}, f\right)$.

1.6. For $\mathscr{P} \subset X \times X, \mathscr{P}^{*} \equiv\{(x, y) \mid(y, x) \in \mathscr{P}\}$. Note $\mathscr{P}^{* *}=\mathscr{P}$.

\section{Attractors and quasi-attractors}

Definition 2.1A. A set $A$ is called an attractor for $f$ if $A$ admits a (closed) neighbourhood $N$ such that $A=\dot{\omega}(N, f)$.

If $\boldsymbol{A}$ is an attractor, $\tilde{\alpha}(A, f)$ is called the domain of influence of $A$.

If $A$ is an attractor for $f^{*}, A$ is called a repeller for $f$ and $\tilde{\alpha}\left(A, f^{*}\right)$ is again called the domain of influence.

Definition 2.1B. An attractor neighbourhood means a closed subset $N$ of $X$ such that for $x \in \partial N, \omega\left(x, f^{*}\right) \subset \operatorname{Ext} N$.

LeмmA 2.2A. If $N$ is an attractor neighbourhood, then the set $A \equiv\{x \mid x \cdot R \subset N\}$ is an attractor and $N \subset \tilde{\alpha}(A, f)$.

Proof. $A$ is closed since it is the maximal invariant set in the closed set $N$. Let $A^{\prime} \equiv \omega(N, f)$. Then $A^{\prime}$ is a closed invariant set containing $A$. For $y \in A^{\prime}$ and any $t$, there exists $t^{\prime} \leq t$ such that $y \cdot t^{\prime} \in N$. Thus $\omega\left(y, f^{*}\right) \cap N \neq \varnothing$. It follows that $y \cdot R \cap$ $\partial N=\varnothing$. Since $y \cdot R \cap N=\varnothing, y \cdot R \subset N$. Thus $A=A^{\prime} . N$ is a neighbourhood of $A$ since $A \cap \partial N=\varnothing$. Thus $A$ is an attractor. For $x \in N, \omega(x, t) \subset \omega(N, f)=A$; thus $N \subset \tilde{\alpha}(A, f)$.

Remark. If $N$ is any closed subset of $X$ such that $x \in \partial N$ implies $\omega(x, f) \cap$ Ext $N \neq \varnothing$, then $A \equiv\{y \mid y \cdot R \subset N\}$ is an attractor. However, it is not generally true that $N \subset \tilde{\alpha}(A, f)$.

THEOREM 2.2B. If $A$ is an attractor, then $A$ is a closed invariant set, $\tilde{\alpha}(A, f)$ is an open invariant set and $A^{*} \equiv \operatorname{Comp} \tilde{\alpha}(A, f)$ is a repeller.

Also $\alpha(A, f)=X \backslash A^{*}$ and $\tilde{\alpha}\left(A^{*}, f^{*}\right)=X \backslash A$ while $A^{* *}=A$.

Proof. That $A$ is closed and invariant and that $\tilde{\alpha}(A, f)$ and $A^{*}$ are invariant is clear.

Let $A=\omega(N, f)$ where $N$ is a neighbourhood of $A$. Then $N \subset \tilde{\alpha} \equiv \alpha(A, f)$, so the latter is a neighbourhood of $A$. Now $\partial \tilde{\alpha}$ is a closed invariant set and thus contains the $\omega$-limit set of each of its points. Furthermore, $\partial \tilde{\alpha} \cap A=\varnothing$. Thus $\partial \tilde{\alpha} \cap \tilde{\alpha}=\varnothing$ and $\tilde{\alpha}$ is open. Observe also that if $x \in \tilde{\alpha}$, then $x \cdot R$ meets $N$ so that $\omega(x, f) \subset \omega(N, f)=A$.

Now $A^{*}=$ Comp $\tilde{\alpha}$ is a closed invariant set. Let $U$ be any open neighbourhood of $A$ with $\mathrm{Cl} U \subset N$ and let $N^{*}=\operatorname{Comp} U$. Then for $x \in \partial N^{*}=\partial U \subset \tilde{\alpha}(A, f)$, $\omega(x, f) \subset A \subset \operatorname{Ext} N^{*}$. Thus $N^{*}$ is an attractor neighbourhood for $f^{*}$. The set $\left\{x \mid x \cdot R \subset N^{*}\right\}$ is disjoint from $\tilde{\alpha}(A, f)$ but must contain all points in $A^{*}=$ Comp $\tilde{\alpha}(A, f)$. Thus $A^{*}$ is an attractor for $f^{*}$.

For $x \in \tilde{\alpha}(A, f) \backslash A, \omega\left(x, f^{*}\right) \subset$ Ext $N \subset$ Int $N^{*}$; thus $\omega\left(x, f^{*}\right) \subset A^{*}$ and $x \in$ $\tilde{\alpha}\left(A^{*}, f^{*}\right)$. If follows that $\tilde{\alpha}\left(A^{*}, f^{*}\right)=X \backslash A$ and the theorem is proved. 
Corollary 2.2C. Any closed neighbourhood $N$ of $A$ which is contained in $\tilde{\alpha}(A, f)$ is an attractor neighbourhood.

Proof. If $x \in \partial N$, then $\omega\left(x, f^{*}\right) \subset A^{*} \subset$ Ext $N$.

Remark. $\phi$ and $X$ are both attractors and repellers with neighbourhoods $\phi$ and $X$ respectively.

Theorem 2.2D. If $A_{1}$ and $A_{2}$ are attractors, then $A=A_{1} \cap A_{2}$ and $\bar{A}=A_{1} \cup A_{2}$ are attractors. Also $A^{*}=A_{1}^{*} \cup A_{2}^{*}, \bar{A}^{*}=A_{1}^{*} \cap A_{2}^{*}, \tilde{\alpha}(A, f)=\tilde{\alpha}\left(A_{1}, f\right) \cap \tilde{\alpha}\left(A_{2}, f\right)$ and $\tilde{\alpha}(\bar{A}, f)=\tilde{\alpha}\left(A_{1}, f\right) \cup \tilde{\alpha}\left(A_{2}, f\right)$.

Proof. Let $N_{1}$ and $N_{2}$ be attractor neighbourhoods for $A_{1}$ and $A_{2}$. Then $N_{1} \cap N_{2}$ is obviously an attractor neighbourhood and $A_{1} \cap A_{2}$ is the corresponding attractor.

Since $\tilde{\alpha}\left(A_{\beta}, f\right)=\left\{y \mid \omega(y, f) \subset A_{\beta}\right\}$ for $\beta=1,2$ (in contrast to the general definition), it is clear that $\tilde{\alpha}\left(A_{1} \cap A_{2}, f\right)=\tilde{\alpha}\left(A_{1}, f\right) \cap \tilde{\alpha}\left(A_{2}, f\right)$. If follows that $A^{*}=$ Comp $\tilde{\alpha}(A, f)$ is given by $A_{1}^{*} \cup A_{2}^{*}$. Applying the above argument with $A_{1}^{*}, A_{2}^{*}$ and $f^{*}$ replacing $A_{1}, A_{2}$ and $f$, the theorem follows.

Definition 2.3A. An invariant set of $A$ which is the intersection of attractors will be called a quasi-attractor. A quasi-repeller of $f$ means a quasi-attractor of $f^{*}$.

Remark. Attractors are isolated invariant sets ([2], [5]). Thus quasi-attractors are quasi-isolated invariant sets ([5]). The only sets which are both attractors and repellers are $X$ and $\phi$; however, it is possible to have non-trivial sets which are both quasi-attractors and quasi-repellers.

Remark. For any subset $Y \subset X$, there is a unique smallest quasi-attractor containing $Y$.

THEOREM 2.3B. Let $A$ be a closed invariant set of $f$. Then $A$ is a quasi-attractor if and only if any neighbourhood of $A$ contains an attractor neighbourhood, while $A$ is an attractor if and only if every small enough closed neighbourhood of $A$ is an attractor neighbourhood.

Proof. This follows immediately from $2.2 \mathrm{C}$ and $2.3 \mathrm{~A}$.

THEOREм 2.4A. Any closed-open subset of an attractor is an attractor. Any component of a quasi-attractor is a quasi-attractor.

Proof. A component of a quasi-attractor must be the intersection of components of attractors and thus of closed-open subsets of attractors. Thus the second statement follows from the first.

If $A^{\prime} \subset A$ is a closed-open subset of the attractor $A$, then there is a closed neighbourhood $N$ of $A^{\prime}$ such that $N \subset \tilde{\alpha}(A, f)$ and $N \cap A=A^{\prime}$. Thus $N$ is an attractor neighbourhood $\left(x \in \partial N\right.$ implies $\left.\omega\left(x, f^{*}\right) \subset A^{*} \subset \operatorname{Ext} N\right)$ and so $A^{\prime}=$ $\{x \mid x \cdot R \subset N\}$ is an attractor.

2.4B. It is not generally true that a component of an attractor is an attractor; however:

LeMMA. Let $x$ be a point in an attractor $A$ such that any neighbourhood of $x$ meets at least two components of $A$. Then no neighbourhood of $x$ with closure in $\tilde{\alpha}(A, f)$ is connected. 
In particular, $X$ is not locally connected at $X$.

Proof. Let $Y$ be a connected set with $\mathrm{Cl} Y \subset \tilde{\alpha}(A, f)$. Then $\omega(Y, f)$ is a connected set in $A$ and meets each component of $A$ that $Y$ does. Thus $Y$ meets only one component of $A$ and so cannot be a neighbourhood of $x$.

THEOREM 2.4C. If $X$ is locally connected, each attractor has finitely many components and each component is an attractor.

Proof. This is from $2.4 \mathrm{~A}$ and $2.4 \mathrm{~B}$.

Remark. It is not true that attractors in a locally connected space have to be locally connected.

THEOREM 2.4D. Suppose $A$ is an attractor for $f$ and that $\bar{A} \subset A$ is an attractor for $\bar{f} \equiv f \mid A$. Then $\bar{A}$ is an attractor for $f$.

Consequently if $\bar{A}$ is a quasi-attractor for $\bar{f} \equiv f \mid A$, where $A$ is a quasi-attractor for $f$, then $\bar{A}$ is a quasi-attractor for $f$.

Proof. Choose a closed neighbourhood $N$ of $\bar{A}$ relative to $X$ such that $N \subset \tilde{\alpha}(A, f)$ and $N \cap A \subset \tilde{\alpha}(\bar{A}, \bar{f})$. For example, let $N$ be the closed $\varepsilon / 2$ neighbourhood of $\bar{A}$, where $\varepsilon=\min \{\rho(A, X \backslash \tilde{\alpha}(A, f) ; \quad \rho(\bar{A}, A \backslash \tilde{\alpha}(\bar{A}, \bar{f})\}$. Then for $x \in \partial N$, either $x \in \tilde{\alpha}(A, f) \backslash A$ or $x \in A \cap \tilde{\alpha}(\bar{f}, \bar{A})$. In the first case $\omega\left(x, f^{*}\right) \subset \operatorname{Ext} \tilde{\alpha}(A, f) \subset \operatorname{Ext} N$, while in the second case $\omega\left(x, f^{*}\right) \subset A \cap \operatorname{Ext} \tilde{\alpha}(\bar{A}, \bar{f}) \subset$ Ext $N$. Thus $N$ is an attractor neighbourhood (in $X$ ) and the theorem follows.

THEOREM 2.4E. Suppose $X^{\prime}$ is any closed invariant set of $f$ and that $A$ is an attractor (quasi-attractor) for $f$; then $A^{\prime} \equiv A \cap X^{\prime}$ is an attractor (quasi-attractor) for $f^{\prime}=f \mid X^{\prime}$.

Proof. Let $N$ be an attractor neighbourhood of $A$. Then $N \cap X^{\prime}$ is an attractor neighbourhood of $\boldsymbol{A}^{\prime}$.

\section{The chain recurrent set and $\Omega$-limit sets}

\section{1. $(\varepsilon, t, f)$-chains}

Definition 3.1A. Given $(x, y) \in X \times X$ and $\varepsilon, t>0$, an $(\varepsilon, t, f)$-chain from $x$ to $y$ means a collection $\left(x=x_{1}, \ldots, x_{n+1}=y ; t_{1}, \ldots, t_{n}\right)$ such that for $1 \leq i \leq n, t_{i} \geq t$ and $\rho\left(x_{i} \cdot t_{i}, x_{i+1}\right) \geq \varepsilon$.

LемMA 3.1B. If there are $(\varepsilon, t, f)$-chains from $x$ to $y$ and from $y$ to $z$, there is an $(\varepsilon, t, f)$-chain from $x$ to $z$.

Proof. The two given chains are put together in the obvious way.

3.2. The partial order $\mathscr{P}(f)$

Definition 3.2A. $\mathscr{P}(f) \equiv\{(x, y) \mid$ for any $\varepsilon, t>0$, there exists an $(\varepsilon, t, f)$-chain from $x$ to $y$.

THEOREM 3.2B. $\mathscr{P}(f)$ is a transitive relation.

Proof. From 3.1B. 
LEMMA 3.2C. If $(x, y) \in \mathscr{P}(f)$, then for any $\bar{t},(x \cdot \bar{t}, y) \in \mathscr{P}(f)$.

Proof. Given $\varepsilon, t>0$, construct an $(\varepsilon, t+|\bar{t}|, f)$-chain from $x$ to $y$. On replacing $x_{1}=x$ by $\bar{x}_{1}=x \cdot \bar{t}$ and $t_{1}$ by $\bar{t}_{1}=t_{1}-\bar{t}(>t)$, there results an $(\varepsilon, t, f)$-chain from $x \cdot \bar{t}$ to $y$.

Theorem 3.2D. $\mathscr{P}(f)^{*}=\mathscr{P}\left(f^{*}\right)$ (cf. $\S 1.6$ ).

Proof. Suppose $(x, y) \in \mathscr{P}(f)$ and that $\varepsilon, t>0$ are given. Choose $\varepsilon^{\prime} \leq \varepsilon$ such that for $\rho(y, z)<\varepsilon^{\prime}, \rho(y \cdot(-t), z \cdot(-t))<\varepsilon$. Then if $\left(x=x_{1}, x_{2}, \ldots, x_{n+1}=y ; t_{1}, \ldots, t_{n}\right)$ is an $\left(\varepsilon^{\prime}, 2 t, f\right)$-chain from $x$ to $y$, it is easily verified that $\left(y=x_{n+1} ; x_{n} \cdot\left(t_{n}-t\right)\right.$, $\left.x_{n-1} \cdot t_{n-1}, \ldots, x_{1} \cdot t_{1}, x ; t, t_{n}-t, t_{n-1}, \ldots, t_{1}\right)$ is an $\left(\varepsilon, t, f^{*}\right)$-chain from $y$ to $x$. (An initial orbit segment has been attached to $y$; otherwise the chain just goes backward over the given one.)

THEOREM 3.2E. $\mathscr{P}(f)$ is closed in $X \times X$.

Proof. Suppose $(x, y)$ is a limit point of $\mathscr{P}(f)$ and let $\varepsilon, t>0$ be given. Using 3.2C, $(x \cdot t, y)$ is also a limit point of $\mathscr{P}(f)$. Choose $\left(x^{\prime}, y^{\prime}\right) \in \mathscr{P}(f)$ such that $\rho\left(\left(x^{\prime}, y^{\prime}\right)\right.$, $(x \cdot t, y))<\varepsilon / 2$. Choose an $(\varepsilon / 2, t, f)$-chain from $x^{\prime}$ to $y^{\prime}$. On replacing $x_{n+1}=y^{\prime}$ by $\bar{x}_{n+1}=y$, an $(\varepsilon, t, f)$-chain from $x^{\prime}$ to $y$ is obtained. Also $\left(x=x_{1}, x_{2}=x^{\prime} ; t_{1}=t\right)$ is an $(\varepsilon, t, f)$-chain from $x$ to $x^{\prime}$. By 3.1B $x$ and $y$ are connected by an $(\varepsilon, t, f)$-chain and the theorem follows.

TheOREM 3.2F. If $(x, y) \in \mathscr{P}(f)$, then $\mathrm{Cl}(x \cdot R) \times \mathrm{Cl}(y \cdot R) \subset \mathscr{P}(f)$.

Proof. Apply 3.2C, 3.2D, 3.2C, 3.2D and 3.2E.

Theorem 3.2G. If $x \in \omega\left(y, f^{*}\right)$, then $(x, y) \in P(f)$.

If $y \in \omega(x, f)$, then $(x, y) \in \mathscr{P}(f)$ and $(x, z) \in \mathscr{P}(f)$ if and only if $(y, z) \in \mathscr{P}(f)$.

Proof. Suppose $x \in \omega\left(y, f^{*}\right)$ and let $\varepsilon, t>0$ be given. Choose $t^{\prime}>t$ such that $\rho\left(x \cdot t, y \cdot\left(-t^{\prime}\right)\right)<\varepsilon$. Then $\left(x=x_{1}, x_{2}=y \cdot\left(-t^{\prime}\right), x_{3}=y ; t_{1}=t, t_{2}=t^{\prime}\right)$ is an $(\varepsilon, t, f)$ chain from $x$ to $y$.

Suppose $y \in \omega(x, f)$. Given $(\varepsilon, t)>0$, choose $t^{\prime}>t$ so that $\rho\left(x \cdot t^{\prime}, y\right)<\varepsilon$. Then $\left(x, y ; t^{\prime}\right)$ is the required chain from $x$ to $y$.

If $(y, z) \in \mathscr{P}(f), 3.2 \mathrm{~B}$ implies $(x, z)$ is. If $(x, z) \in \mathscr{P}(f)$ and $\varepsilon, t>0$ are given, choose $\bar{t}$ so that $\rho(x \cdot \bar{t}, y \cdot t)<\varepsilon$, By $3.2 \mathrm{~F}(x \cdot \bar{t}, z) \in \mathscr{P}(f)$. Also $(y, x \cdot \bar{t} ; t)$ is an $(\varepsilon, t, f)$-chain from $y$ to $x \cdot \bar{t}$. Thus there is an $(\varepsilon, t, f)$-chain from $y$ to $z$ and the theorem follows.

\subsection{The chain recurrent set and an equivalence relation}

Definition 3.3A. Let $R(f) \equiv\{x \mid(x, x) \in \mathscr{P}(f)\} ; R(f)$ will be called the chain recurrent set.

Let $\mathscr{E}(f) \equiv \mathscr{P}(f) \cap \mathscr{P}(f)^{*}$.

THEOREM 3.3B. $R(f)$ is a closed invariant set of $f$ containing the non-wandering set and $R(f)=R\left(f^{*}\right)$.

$\mathscr{E}(f)=\mathscr{E}\left(f^{*}\right) \subset R(f) \times R(f)$ is closed in $R(f) \times R(f)$ (hence in $X \times X$ ) and is an equivalence relation. 
Proof. $R(f)$ is closed and invariant by $3.2 \mathrm{E}$ and $3.2 \mathrm{~F}$ and equals $R\left(f^{*}\right)$ by 3.2D. $\mathscr{E}(f)=\mathscr{E}\left(f^{*}\right)$ by $3.2 \mathrm{D}$ and is closed by $3.2 \mathrm{E}$. If $(x, y) \in \mathscr{E}(f)$, then both $x$ and $y$ are in $R(f)$. If $x \in R(f)$, then $(x, x) \in \mathscr{P}(f)$ so in $\mathscr{E}(f)$. $\mathscr{E}(f)$ is symmetric by definition and transitive since $\mathscr{P}(f)$ is. Thus $\mathscr{E}(f)$ is an equivalence relation on $R(f)$. If $x$ is a non-wandering point and $\varepsilon, t>0$, then an $(\varepsilon, t, f)$-chain from $x$ to $x$ is easily constructed.

THEOREM 3.3C. The equivalence classes of $\mathscr{E}(f)$ are unions of components of $R(f)$ and each meets at most one component of $X$ non-trivially.

Proof. Let $R^{\prime}$ be a component of $R$ and suppose $x, y \in R^{\prime}$. Then, since $R^{\prime}$ is closed, given $(\varepsilon, t)>0$, there is a finite sequence $\left(x=x_{1}, \ldots, x_{n+1}=y\right)$ in $R$ such that $\rho\left(x_{i}, x_{i+1}\right)<\varepsilon / 2$. Since $\left(x_{i}, x_{i}\right) \in \mathscr{P}(f)$, there is an $(\varepsilon / 2, t, f)$-chain from $x_{i}$ to $x_{i}$. Replacing the last point of this chain by $x_{i+1}$, one has an $(\varepsilon, t, f)$-chain from $x_{i}$ to $x_{i+1}$. If follows that such a chain exists from $x$ to $y$. Since $x$ and $y$ were arbitrary points of $R^{\prime}$, all points of $R^{\prime}$ are equivalent.

If $(x, y) \in \mathscr{E}(f)$, then the $(\varepsilon, t, f)$-chains from $x$ to $y$ show that $x$ and $y$ are ' $\varepsilon$-connected' in $X$ for any $\varepsilon$. Thus $x$ and $y$ lie in the same component of $X$.

Remark. This does not imply $x$ and $y$ are in the same component of $R(f)$ since the chains may not be contained in $R(f)$. However, see 3.6D.

\section{4. $\Omega$-limit sets}

Definition. 3.4A. For $C \subset X, \Omega(C, f) \equiv\{y \mid$ there exists $x \in C$ with $(x, y) \in \mathscr{P}(f)\}$. Note $\Omega(C, f)=\bigcup_{x \in C} \Omega(x, f)$.

THEOREM 3.4B. If $C$ is closed, $\Omega(C, f)$ is a closed invariant set.

If $\left\{C_{n}\right\}_{1}^{\infty}$ is a sequence of closed subsets of $X$ and $C=\lim \sup \left\{C_{n}\right\}_{1}^{\infty}$, then $\Omega(C, f) \supset$ $\lim \sup \left\{\Omega\left(C_{n}, f\right)\right\}_{1}^{\infty}$.

Proof. $\Omega(C, f)$ is invariant by $3.2 \mathrm{~F}$ and the rest follows from $3.2 \mathrm{E}$.

LEMMA 3.4C. If $C$ is closed, $\Omega(C, f) \supset \omega(C, f)$.

Proof. Suppose $y \in \omega(C, f)=\bigcap_{t \in R} \mathrm{Cl}\{C \cdot(t, \infty)\}$. Then given $n>0$, there exists $x_{n} \in C$ such that $\rho\left(x_{n} \cdot n, y\right)<1 / n$. Let $x \in C$ be a limit point of $\left\{x_{n}\right\}_{1}^{\infty}$. Given $\varepsilon$, $t>0$, choose $\varepsilon^{\prime}$ between 0 and $\varepsilon$ so that $\rho\left(x^{\prime}, x\right)<\varepsilon^{\prime}$ implies $\rho\left(x^{\prime} \cdot t, x \cdot t\right)<\varepsilon$. Choose $n$ so that $n>2 t, 1 / n<\varepsilon$ and $\rho\left(x_{n}, x\right)<\varepsilon^{\prime}$. Then $\left(x=x_{1}, x_{2}=x_{n} \cdot t, x_{3}=y\right.$; $\left.t_{1}=t, t_{2}=n-t\right)$ is an $(\varepsilon, t, f)$-chain from $x$ to $y$. Thus $(x, y) \in \mathscr{P}(f)$ and $y \in \Omega(C, f)$.

THEOREM 3.4D. If $C$ is closed, then $\Omega(C, f)=\Omega(\omega(C, f), f)$.

Proof. By 3.2G $\Omega(x, f)=\Omega(\omega(x, f), f)$. Thus $\Omega(C, f)=\bigcap_{x \in C} \Omega(\omega(x, f), f)$. Since $\omega(x, f) \subset \omega(C, f)$ whenever $x \in C, \Omega(c, f) \subset \Omega(\omega(C, f), f)$. But by $3.4 \mathrm{C}$ and $3.2 \mathrm{~B}$ $\Omega(C, f) \supset \Omega(\omega(C, f), f)$ and the theorem follows.

Theorem 3.4E.

(1) $y \in \Omega(x, f)$ if and only if $x \in \Omega\left(y, f^{*}\right)$.

(2) If $(x, y) \in \mathscr{E}(f)$, then $\Omega(x, f)=\Omega(y, f)$. 
(3) $\Omega(x, f) \cap \Omega\left(x, f^{*}\right)$ is non-empty if and only if $x \in R(f)$; in this case it is precisely the equivalence class of $x$.

(4) For $x \in R(f), \omega(x, f) \cup \omega\left(x, f^{*}\right) \subset \Omega(x, f) \cap \Omega\left(x, f^{*}\right)$.

(5) In any case $\Omega(x, f)$ contains any component of $R(f)$ which it meets non-trivially.

Proof. The first statement comes from 3.2D; the second and third from 3.2B and the definitions. The fourth comes from $3.2 \mathrm{G}$ and the last from $3.3 \mathrm{C}$.

3.5. The $(\varepsilon, t)$-approximation of the $\Omega$-limit set

Definition 3.5A. $\Omega^{\prime}(C, \varepsilon, t, f) \equiv\{y \mid$ there is an $(\varepsilon, t, f)$-chain from $x$ to $y$ for some $x \in C\}$. Note that if $\bar{C} \subset C$, then $\Omega^{\prime}(\bar{C}, \varepsilon, t, f) \subset \Omega^{\prime}(C, \varepsilon, t, f)$.

LEMMA 3.5B. $\Omega^{\prime}(C, \varepsilon, t, f)$ is open and contains $\Omega(C, f)$.

Proof. Given an $(\varepsilon, t, f)$-chain from $x$ to $y$, let $\varepsilon^{\prime} \equiv \rho\left(x_{n} \cdot t_{n}, y\right)$. For $z$ such that $\rho(z, y)<\varepsilon-\varepsilon^{\prime}$, an $(\varepsilon, t, f)$-chain from $x$ to $z$ is obtained on replacing $x_{n+1}=y$ by $\bar{x}_{n+1}=z$. It follows that $\Omega^{\prime}(C, \varepsilon, t, f)$ is open.

LEMMA 3.5C. The closure of $\Omega^{\prime}(C, \varepsilon, t, f)$ is an attractor neighbourhood and contains an $\varepsilon$-neighbourhood of the corresponding attractor.

Proof. If $y \in \Omega^{\prime} \equiv \Omega^{\prime}(C, \varepsilon, t, f)$ and $\rho(z, y \cdot t)<\varepsilon$, then $(y, z ; t)$ is an $(\varepsilon, t, f)$-chain from $y$ to $z$ and so $z \in \Omega^{\prime}$. For $y \in \partial \Omega^{\prime}$ choose a neighbourhood $U$ of $\omega\left(y, f^{*}\right)$ such that for $z \in U, \rho\left(z \cdot t, \omega\left(y, f^{*}\right)\right)<\varepsilon$. Then each $z \in U$ is connected to $\omega\left(y, f^{*}\right)$ and thence to $y$ by an $(\varepsilon, t, f)$-chain. Since $y \in \partial \Omega^{\prime}, y$ is not in the open set $\Omega^{\prime}$. Thus $U \cap \Omega^{\prime}=\varnothing$ and $\omega\left(y, f^{*}\right) \subset \operatorname{Ext} \Omega^{\prime}$ so that $\mathrm{Cl} \Omega^{\prime}$ is an attractor neighbourhood.

If $\Omega^{\prime} \supset Y$, then the first sentence of the proof implies that an $\varepsilon$-neighbourhood of $Y \cdot t$ is in $\Omega^{\prime}$. For $A$ the attractor in $\mathrm{Cl}^{\prime}, A=A \cdot t$; thus the lemma is proved.

Definition 3.5D. $\Omega(C, \varepsilon, t, f) \equiv\left\{x \mid x \cdot R \subset \Omega^{\prime}(C, \varepsilon, t, f)\right\}$.

THEOREM. $\Omega \equiv \Omega(C, \varepsilon, t, f)$ is an attractor containing $\Omega(C, f)$. $\tilde{\alpha}(\Omega, f)$ contains an $\varepsilon$-neighbourhood of $\Omega$; in particular, at most a finite number of the sets $\Omega(C, \varepsilon, t, f)$ are distinct.

Proof. Except for the assertion concerning the number of distinct sets, this is a rewording of $3.5 \mathrm{~B}$ and $3.5 \mathrm{C}$. To see the last, observe that the Hausdorff distance between any two such sets is at least $\varepsilon$. The result then follows from the fact that the space of closed subsets of $X$ with the Hausdorff metric is compact.

LeMmA 3.5E. Given an attractor $A$ and a neighbourhood $U \supset A$, there exist $\bar{\varepsilon}, \bar{t}>0$ such that for $\varepsilon<\bar{\varepsilon}$ and $t \geq \bar{t}, \Omega^{\prime}(A, \varepsilon, t, f) \subset U$. Then with $U \subset \tilde{\alpha}(A, f), \Omega(A, \varepsilon, t, f)=$ A.

Proof. Choose a neighbourhood $N$ of $A$ such that $N \subset U$ and $A=\omega(N, f)$. Then there exists $\bar{t}$ such that $\bar{N} \equiv \operatorname{Cl}\{N \cdot[\bar{t}, \infty)\} \subset$ Int $N$. Let $\bar{\varepsilon}=\rho(\partial N, \bar{N})$. Given $x \in N$, $\varepsilon<\bar{\varepsilon}$ and $t \geq \bar{t}$, let $\left(x=x_{1}, \ldots, x_{n+1}=y ; t_{1}, \ldots, t_{n}\right)$ be an $(\varepsilon, t, f)$-chain from $x$ to $y$. If $x_{i} \in N$, then $x_{i} \cdot t_{i} \in \bar{N}$ so $x_{i+1} \in N$. Since $x_{1} \in N$, it follows that $x_{n+1}=y \in N$ and the result follows. 


\subsection{Characterization of $\Omega$-limit sets}

THEOREM 3.6A. $\Omega(C, f)$ is precisely equal to the smallest quasi-attractor of $f$ containing $\omega(C, f)$. In particular, if $\omega(C, f)$ is connected, then so is $\Omega(C, f)$.

Proof. From the definitions $\Omega(C, f)=\bigcap_{\varepsilon, t>0} \Omega^{\prime}(C, \varepsilon, t, f)$. Thus $\Omega(C, f)$ contains the quasi-attractor $\bigcap_{\varepsilon, t>0} \Omega(C, \varepsilon, t, f)$. On the other hand, since $\Omega(C, f)$ is a closed invariant set in $\Omega^{\prime}(C, \varepsilon, t, f)$, it must be contained in $\Omega(C, \varepsilon, t, f)$. Thus it is equal to the intersection of the latter and so is a quasi-attractor. By $3.4 \mathrm{D}$ it contains $\omega(C, f)$ and by $3.5 \mathrm{E}$ it is contained in any attractor containing $\omega(C, f)$. Since components of quasi-attractors are quasi-attractors $(2.4 \mathrm{~A})$, the connectedness of $\omega(C, f)$ implies that of $\Omega(C, f)$.

Corollary 3.6B. Comp $R(f)=\cup\{\tilde{\alpha}(A, f) \backslash A \mid A$ is an attractor of $f\}$.

Proof. If $A$ is an attractor and $x \in \tilde{\alpha}(A, f) \backslash A$, then $\omega(x, f) \subset A$ and by 3.6A $\Omega(x, f) \subset$ $A$, so that $x \notin \Omega(x, f)$ and $x \in \operatorname{Comp} R(f)$. If $x \notin \Omega(x, f)$, then there is some attractor $A$ containing $\Omega(x, \bar{f})$ but not $x$. Then since $\omega(x, f) \subset \Omega(x, f) \subset A, x \in \tilde{\alpha}(A, f)$.

THEOREM 3.6C. Let $A$ be a quasi-attractor off and let $\bar{f}=f \mid A$. Then $\Omega(x, \bar{f})=\Omega(x, f)$ for any $x \in A$.

Let $A^{*}$ be a quasi-repeller of $f$ and let $\bar{f}=f \mid A^{*}$. Then $\Omega(x, \bar{f})=A^{*} \cap \Omega(x, f)$ for any $x \in A^{*}$.

Proof. In the first case $2.4 \mathrm{D}$ implies $\Omega(x, \bar{f})$ is not only a quasi-attractor of $\bar{f}$ but also of $f$. Since it contains $\omega(x, f)$ and is contained in $\Omega(x, f)$, it must be equal to $\Omega(x, f)$ by $3.6 \mathrm{~A}$.

In the second case $2.4 \mathrm{E}$ implies that $A^{*} \cap \Omega(x, f)$ is a quasi-attractor of $\bar{f}$ containing $\omega(x, f)$ hence $\Omega(x, \bar{f})$ (by 3.6A). Let $\bar{A}^{*}$ be any repeller of $\bar{f}$ dual to an attractor containing $\Omega(x, \bar{f})$. By $2.4 \mathrm{D} \bar{A}^{*}$ is also a repeller for $f$. Then its dual attractor (with respect to $f$ ) must contain $\Omega(x, f)$ hence $\Omega(x, f) \cap A^{*}$. Thus any attractor of $\bar{f}$ containing $\Omega(x, \bar{f})$ also contains $\Omega(x, f) \cap A^{*}$ and so $\Omega(x, \bar{f})=\Omega(x, f) \cap A^{*}$.

THEOREM 3.6D. The equivalence classes of $R(f)$ are precisely the components of $R(f)$.

If $\hat{f} \equiv f \mid R(f)$, then $\mathscr{P}(\hat{f})=\mathscr{P}(f)$; thus $(\varepsilon, t, f)$-chains connecting points of $R(f)$ can be chosen to lie in $R(f)$.

Proof. Let $E(x)=\Omega(x, f) \cap \Omega\left(x, f^{*}\right)$ be the equivalence class of $x$. Let $\bar{f} \equiv f \mid \Omega(x, f)$ and let $\bar{f} \equiv \bar{f} \mid E(x)$. By the first sentence of 3.6C $\Omega(x, \bar{f})=\Omega(x, f)$ (note $x \in \Omega(x, f)$ ). By 2.4E $E(x)=\Omega\left(x, f^{*}\right) \cap \Omega(x, f)$ is a quasi-repeller of $\bar{f}$. Thus by $3.6 \mathrm{C}$ (second sentence) $\Omega(x, \bar{f})=E(x) \cap \Omega(x, \bar{f})=E(x)$.

By 3.6A $E(x)$ is connected and so is a component of $R(f)$ (using 3.3C). In a similar way $E(x)$ is equal to $\Omega\left(x, \bar{f}^{*}\right)$. Since $\bar{f}$ chains are also $\hat{f}$ chains, all points of $E(x)$ are equivalent under $\hat{f}$ and, since equivalence classes of $\mathscr{E}(\hat{f})$ are contained in components of $R(\hat{f})$, it follows that $E(x)$ is an equivalence class of $\mathscr{E}(\hat{f})$. 


\section{The gradient part of $f$}

\subsection{Functions which decrease on orbits}

Definition 4.1A. Given a flow $f$ on $X$, define:

$\mathscr{D}^{\prime}(f) \equiv\{g: X \rightarrow R \mid g$ is continuous and for $x \in X$ and $t>0, g(x \cdot t) \leq g(x)\}$.

$R^{\prime}(f) \equiv\left\{x \in X \mid\right.$ for $g \in \mathscr{D}^{\prime}(f)$ there exists $t>0$ such that $\left.g(x \cdot t)=g(x)\right\}$.

$\mathscr{D}(f) \equiv\left\{g \in \mathscr{D}^{\prime}(f) \mid\right.$ if $x \notin R(f)$ and $t>0$, then $\left.g(x \cdot t)<g(x)\right\}$.

4.1B. These definitions are obtained from Auslander [1], who was interested in a more general situation. The set $R^{\prime}(f)$ is called the generalized recurrent set by Auslander and he justifies the name by characterizing the points of $R^{\prime}(f)$ in terms of orbit prolongations. Our lemma $4.1 \mathrm{E}$ below is of the same type as his result (though easier as well as more suited to the present purpose). Lemmas $4.1 \mathrm{C}$ and 4.1D are also proved by him in his more general setting.

LEMMA 4.1C. $R^{\prime}(f)$ is a closed invariant set.

Proof. $R^{\prime}(f)$ is the intersection of the closed sets $C(g, t)$ defined for $g \in \mathscr{D}^{\prime}(f)$ and $t>0$ by $C(g, t) \equiv\{x \mid g(x \cdot t)-g(x)=0\}$. Thus $R^{\prime}(f)$ is closed. For $x \notin R^{\prime}(f)$ there exists $g \in \mathscr{D}^{\prime}(f)$ such that for $t>0, g(x \cdot t)<g(x)$. Given $\bar{t} \in R$, let $\bar{g}$ be defined by $\bar{g}(x)=g(x \cdot(-\bar{t}))$. Then $\bar{g} \in \mathscr{D}^{\prime}$ and for $t>0, \bar{g}((x \cdot \bar{t}) \cdot t)=g(x \cdot t)<g(x)=\bar{g}(x \cdot \bar{t})$. Thus $x \notin R^{\prime}(f)$ implies $x \cdot \bar{t} \notin R(f)$ for all $\bar{t} \in R$ and so Comp $R^{\prime}(f)$ and $R^{\prime}(f)$ are invariant.

LEMMA 4.1D. $\mathscr{D}^{\prime}(f)$ is a complete metric space (under the sup norm metric or equivalently the compact-open topology) and $\mathscr{D}(f)$ contains a dense set of second Baire category in $\mathscr{D}^{\prime}(f)$. In particular, $\mathscr{D}(f) \neq \varnothing$.

Proof. $\mathscr{D}^{\prime}(f)$ is a closed subset of the space of continuous functions on $X$ with the sup norm metric. Thus $\mathscr{D}^{\prime}(f)$ is a complete metric space.

If $R^{\prime}(f)=X$, then $\mathscr{D}^{\prime}(f)=\mathscr{D}(f)$ and the lemma is true.

If not, let $m$ be a fixed positive integer. For $x \in \operatorname{Comp} R^{\prime}(f)$ choose a function $g \in \mathscr{D}^{\prime}(f)$ such that $g(x \cdot 1 / m)<g(x)$. Since $g$ is continuous, there is a compact neighbourhood $C(x)$ of $x$ such that $g|C(x)>g| C(x) \cdot 1 / m$ (pointwise).

Thus there is obtained a cover of Comp $R^{\prime}(f)$ from which a countable sub-cover $\left\{C_{n}\right\}_{1}^{\infty}$ can be chosen.

Now for each positive integer $n$, let $\phi(n, m)$ be the set $\left\{g \in \mathscr{D}^{\prime}(f)|g| C_{n}>\right.$ $\left.g \mid C_{n} \cdot 1 / m\right\}$. Since $C_{n}$ is compact, $\phi(n, m)$ is open in $\mathscr{D}^{\prime}(f)$; it is non-empty by the construction of the $C_{n}$. For $\bar{g} \in \mathscr{D}^{\prime}(f)$ and $\varepsilon>0, g \in \phi(n, m)$ implies $\bar{g}+\varepsilon g \in \phi(n, m)$. Thus $\phi(n, m)$ is also dense in $\mathscr{D}^{\prime}(f)$ so $\bigcap_{n=1}^{\infty} \phi(n, m)$ is a dense set of second Baire category with the property that for $g$ in this set and $x \in \operatorname{Comp} R^{\prime}(f), g(x \cdot 1 / m)<$ $g(x)$.

Let $\bar{\phi}(m) \equiv\left\{g \mid x \in\right.$ Comp $R^{\prime}(f)$ implies $\left.g(x \cdot 1 / m)<g(x)\right\}$. Then $\bar{\phi}(m)$ contains a dense set of second Baire category as does $\bigcap_{m=1}^{\infty} \bar{\phi}(m)$. But the last set is just $\mathscr{D}(f)$ so the theorem is proved. 
LEMMA 4.1E. $R^{\prime}(f) \subset R(f)$.

Proof. Suppose $\bar{x} \notin R(f)$. By $3.6 \mathrm{~B}$ there is an attractor $A$ such that $\bar{x} \in \tilde{\alpha}(A, f) \backslash A$. Let $A^{*}$ be the dual repeller.

Let $\rho: X \rightarrow[0,1]$ be any continuous function such that $\rho\left|A^{*}=1, \rho\right| A=0$. Define $\bar{\rho}(x)=\sup \{\rho(y) \mid y \in X \cdot[0, \infty)\}$. Clearly $\bar{\rho}$ decreases on orbits so is in $\mathscr{D}^{\prime}(f)$ provided it is continuous.

For $x \in \tilde{\alpha}(A, f) \backslash A$, let $\rho(x)=\delta$. Let $C \subset \tilde{\alpha}(A, f)$ be a compact neighbourhood of $x$ such that $\rho \mid C>\delta / 2$. Choose a neighbourhood $u$ of $A$ such that $g \mid u<\delta / 2$. Choose $t>0$ such that $\mathrm{Cl}\{C \cdot(t, \infty)\} \subset u$. Then for $x^{\prime} \in C, \bar{\rho}\left(x^{\prime}\right)=\sup \left\{\rho(y) \mid y \in x^{\prime}[0, \infty]\right\}=$ $\sup \left\{\rho(y) \mid y \in x^{\prime} \cdot[0, t]\right\}$. Since $\rho$ is continuous and $x^{\prime}[0, t] \rightarrow x \cdot[0, t]$ as $x^{\prime} \rightarrow x, \bar{\rho}$ is continuous at $x$.

Thus $\bar{\rho}$ is continuous at all points of $\tilde{\alpha}(A, f) \backslash A$. It is obviously continuous at points of $A^{*}$ and also at points of $A$, since the positive half-orbit from points near $A$ stays near $A$. Thus $\bar{\rho}: X \rightarrow R$ is continuous and so in $\mathscr{D}^{\prime}(f)$.

Clearly $\bar{\rho}$ decreases at some point of every orbit in $\tilde{\alpha}(A, f) \backslash A$. Thus this set, and in particular $\bar{x}$, is contained in Comp $R^{\prime}(f)$. Thus $\operatorname{Comp} R(f) \subset \operatorname{Comp} R^{\prime}(f)$ and the lemma follows.

Remark 4.1F. It is not generally true that $R^{\prime}(f)=R(f)$. For example, let $X$ denote the unit square in the plane and let $f$ be the flow generated by the vector field $\dot{x}=0$, $\dot{y}=-x y(1-x)(1-y)$. Then $R^{\prime}(f)$ is the boundary of the square (rest points are clearly in $R^{\prime}(f)$ while the function $g(x, y)=y$ decreases on all other orbits) while $R(f)=X$ (there are no non-trivial attractors).

\subsection{Gradient flows}

Definition 4.2A. A flow $f$ will be called a gradient flow if $R(f)$ is totally disconnected. Note. Then $R(f)$ consists of rest points and has point-like components. The example of 4.1F is 'gradient' in the sense that there exists a function which decreases on non-constant orbits, but it admits no filtrations. The point of view here is that the filtration is the notion of importance.

THEOREM 4.2B. If $f$ is gradient-like, $R^{\prime}(f)=R(f)$ and in particular, for $g \in \mathscr{D}(f), g$ is strictly decreasing on all non-constant orbits. (This is meant to justify the name 'gradient flow'.)

Proof. Since $R(f)$ consists of rest points and all rest points are in $R^{\prime}(f), R^{\prime}(f) \supset$ $R(f)$. The reverse inclusion follows from 4.1E. The remainder is from the definitions.

\subsection{Domination}

Definition 4.3A. Let $f$ and $f^{\prime}$ be flows on $X$ and $X^{\prime}$ respectively and let $h$ be a continuous map from $X$ onto $X^{\prime}$. If for each $x \in X, h(x \cdot[0, \infty))=h(x) \cdot[0, \infty)$, then $f$ will be said to dominate $f^{\prime}$ with map $h$. In symbols, $f>f^{\prime}(h)$. If $f>f^{\prime}$ and $f^{\prime}>f$, then $f$ and $f^{\prime}$ will be called ' $\omega$-similar'.

LEMMA 4.3B. The relation > between flows on compact metric spaces is reflexive and transitive. 
Suppose $f>f^{\prime}(h)$. Then if $Y$ is a closed invariant set of $f, h(Y)$ is a closed invariant set of $f^{\prime}$. If $Y$ is a closed invariant set of $f^{\prime}$, then $h^{-1}(Y)$ is a closed invariant set of $f$.

LEMMA 4.3C. If $f>f^{\prime}(h)$, then for any $Y \subset X, h(\omega(Y, f))=\omega\left(h(Y), f^{\prime}\right)$.

Proof. Since $X$ and $X^{\prime}$ are compact and Hausdorff, for any $Y \subset X, h(\mathrm{Cl}(Y))=$ $\mathrm{Cl}(h(Y))$.

From the definition (4.3A), for $t \in R, h(Y \cdot[t, \infty))=h(Y) \cdot[t, \infty)$ (the $\cdot$ is used both for $f$ and $f^{\prime}$ ).

Thus $h\left(\mathrm{Cl}(C \cdot[t, \infty))=\mathrm{Cl}(h(C) \cdot[t, \infty)) \quad\right.$ and $\quad \bigcap_{t \in R} h(\mathrm{Cl}(C \cdot[t, \infty))=$ $\omega\left(h(C), f^{\prime}\right)$.

Since the sets $\mathrm{Cl}(C \cdot[t, \infty))$ are compact and decrease as $t$ increases, and since $X^{\prime}$ is Hausdorff, $\bigcap_{t \in R} h(\mathrm{Cl}(C \cdot[t, \infty)))=h\left(\bigcap_{t \in R} \mathrm{Cl}(C \cdot[t, \infty))\right)=h(\omega(c, f))$. Thus the theorem is proved.

LEMMA 4.3D. Suppose $f>f^{\prime}(h)$.

If $A^{\prime}$ and $A^{\prime *}$ form an attractor-repeller pair for $f^{\prime}$, then $h^{-1}\left(A^{\prime}\right)$ and $h^{-1}\left(A^{\prime *}\right)$ form an attractor-repeller pair for $f$ and $h\left(\tilde{\alpha}\left(h^{-1}\left(A^{\prime}\right), f\right)\right)=\tilde{\alpha}\left(A^{\prime}, f^{\prime}\right)$.

If $A$ and $A^{*}$ form an attractor-repeller pair for $f$, and $h(A) \cap h\left(A^{*}\right)=\varnothing$, then $h(A)$ and $h\left(A^{*}\right)$ form an attractor-repeller pair for $f^{\prime}$ and $h(\tilde{\alpha}(A, f))=\tilde{\alpha}(h(A), f)$.

Proof. Observe that two closed invariant sets $A$ and $A^{*}$ of $f$ form an attractor-repeller pair if and only if they are disjoint and $x \in \operatorname{Comp}\left(A \cup A^{*}\right)$ implies $\omega\left(x, f^{*}\right) \subset A^{*}$ and $\omega(x, f) \subset A$.

If $A^{\prime}$ and $A^{\prime *}$ are an attractor-repeller pair for $f^{\prime}$, then $h^{-1}\left(A^{\prime}\right)$ and $h^{-1}\left(A^{\prime *}\right)$ are closed disjoint invariant sets. Also $x \in \operatorname{Comp}\left(h^{-1}\left(A^{\prime}\right) \cup h^{-1}\left(A^{\prime *}\right)\right)$ implies $h(x) \in$ Comp $\left(A^{\prime} \cup A^{\prime *}\right)$ and an application of $4.3 \mathrm{C}$ shows $h^{-1}\left(A^{\prime}\right)$ and $h^{-1}\left(A^{\prime *}\right)$ form an attractor-repeller pair for $f$.

If $A$ and $A^{*}$ form an attractor-repeller pair for $f$, and $h(A)$ and $h\left(A^{*}\right)$ are disjoint, then $C$ again implies that $h(A)$ and $h\left(A^{*}\right)$ form an attractor-repeller pair for $f$.

THEOREM 4.3E. If $f>f^{\prime}(h)$, then for all $x \in X, h(\Omega(x, f)) \subset \Omega\left(h(x), f^{\prime}\right)$ and $h(R(f)) \subset R\left(f^{\prime}\right)$.

Proof. Since $\Omega(h(x), f)$ is the intersection of attractors containing $\omega\left(h(x), f^{\prime}\right)=$ $h(\omega(x, f))$, and since the inverse image of an attractor is an attractor, $h^{-1}\left(\Omega\left(h(x), f^{\prime}\right)\right)$ obviously contains $\Omega(x, f)$. The result for $R(f)$ follows from that for $\Omega$-limit sets.

THEOREM 4.3F. If $f^{\prime}$ is a gradient flow and $f>f^{\prime}(h)$, then $h(R(f))=R\left(f^{\prime}\right)$.

Proof. If $x^{\prime} \in R\left(f^{\prime}\right)$, then $x^{\prime}$ is a rest point of $f^{\prime}$. It follows that if $h(x)=x^{\prime}$, then $h(\omega(x, f))=x^{\prime}$. Since $\omega(x, f) \subset R(f), h(R(f)) \supset R\left(f^{\prime}\right)$ and by $4.3 \mathrm{E}$ the theorem is proved.

\subsection{The gradient part of $f$}

Definitions and remarks 4.4A. Let $\overline{\mathscr{E}}(f) \equiv \mathscr{E}(f) \cup\{(x, x) \mid x \in X\}$. Then $\overline{\mathscr{E}}(f)$ is an equivalence relation on $X$, each equivalence class being either a point of $\operatorname{Comp} R(f)$ 
or a component of $R(f)$. Also $\overline{\mathscr{E}}(f)$ is closed in $X \times X$ so that the equivalence classes are closed sets comprising an upper semicontinuous decomposition of $X$.

Let $\bar{X}$ be the set of equivalence classes of $\mathscr{E}(f)$ and let $\pi: X \rightarrow \bar{X}$ send each point to its equivalence class. Define a set $u \subset \bar{X}$ to be open if $\pi^{-1}(u)$ is open; then the open sets form a topology on $\bar{X}$ such that $\bar{X}$ is a compact metric space.

LEMMA 4.4B. $\pi(R(f))$ is a totally disconnected subset of $\bar{X}$.

Proof. Let $U \subset X$ be an open set such that $\partial U \cap R(f)=\varnothing$. Suppose $x \in \pi^{-1}(\pi(U))$ and let $y \in U$ be such that $\pi(y)=\pi(x)$. Then either $x=y$ or $x$ and $y$ lie in the same component of $R(f)$. In either case (using $\partial U \cap R(f)=\varnothing$ in the second) $x \in U$. Thus $\pi^{-1}(\pi(U))=U$ and is in particular open. Given two points of $\pi(R(f))$, there correspond two components of $R(f)$. Choose disjoint open sets of $X$ with boundaries in Comp $R(f)$ and such that each contains one of the components. Then the images of these two open sets are disjoint and open in $\bar{X}$ and have boundaries in $\bar{X} \backslash \pi(R(f))$. Also each contains one of the given points in $\pi(R(f))$. Thus $\pi(R(f))$ is totally disconnected.

Definition 4.4.C. For $\bar{x} \in \bar{X}$, define $\hat{f}(\bar{x}, t) \equiv \pi\left(\pi^{-1}(\bar{x}) \cdot t\right)$.

LeMMA. For all $\bar{x}$ and $t$, the set $\hat{f}(\bar{x}, t)$ consists of precisely one point. Also $\bar{x} \in \hat{f}(\bar{x}, t)$ if and only if $\bar{x} \in \pi(R(f))$.

Proof. If $\bar{x} \notin \pi(R(f))$, then $\pi^{-1}(\bar{x})$ so $\pi^{-1}(\bar{x}) \cdot t$ consists of precisely one point which is not in $R(f)$ Then $\pi\left(\pi^{-1}(\bar{x}) \cdot t\right)$ consists of one point which is not equal to $\bar{x}$. If $\bar{x} \in \pi(R(f))$, then $\pi^{-1}(\bar{x})$ is invariant under $f$ so that $\pi^{-1}(\bar{x}) \cdot t=\pi^{-1}(\bar{x})$ and $\hat{f}(\bar{x}, t)=\{\bar{x}\}$.

Definition 4.4D. Define $\bar{f}: \bar{X} \times R \rightarrow \bar{X}$ by $\bar{f}(\bar{x}, t) \in \hat{f}(\bar{x}, t) . \bar{f}$ will be called the gradient part of $f$.

THEOREM. $\bar{f}$ is a gradient flow on $\bar{X}$ with $f>\bar{f}(\pi)$. If $\bar{f}$ is any other gradient flow dominated by $f$, then $\vec{f}$ dominates $\bar{f}$.

In particular, the rest point set of $\bar{f}$ is in one-one correspondence with the components of $R(f)$.

Proof. It follows from the definition that the diagram

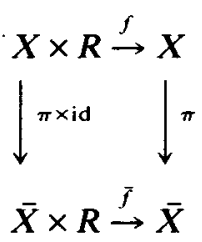

commutes so that, if $\bar{f}$ is a flow, it is dominated by $f$. Again from the definition, $\bar{f}\left(\bar{x}, t_{1}+t_{2}\right)=\bar{f}\left(\bar{f}\left(\bar{x}, t_{1}\right), t_{2}\right)$ and $\bar{f}(\bar{x}, 0)=\bar{x}$ for all $\bar{x}, t_{1}$ and $t_{2}$.

If $\bar{x}_{n} \rightarrow \bar{x}$ and $t_{n} \rightarrow t$, then lim sup $\left\{\pi^{-1}\left(\bar{x}_{n}\right)\right\} \subset \pi^{-1}(\bar{x})$ so that $\lim \sup \left\{\pi^{-1}\left(\bar{x}_{n}\right) \cdot t_{n}\right\} \subset$ $\left\{\pi^{-1}(\bar{x}) \cdot t\right\}$. Thus $\lim \sup \left\{\pi\left(\pi^{-1}\left(\bar{x}_{n}\right) \cdot t_{n}\right)\right\}=\lim \sup \left\{\bar{x}_{n} \cdot t_{n}\right\} \subset \pi\left(\pi^{-1}(\bar{x}) \cdot t\right)=\bar{x} \cdot t$ and, since the latter is a point, $\bar{x}_{n} \cdot t_{n} \rightarrow \bar{x} \cdot t$. Thus $\bar{f}$ is continuous and so a flow. 
Now suppose $A$ and $A^{*}$ form an attractor-repeller pair for $f$. Since no component of $R(f)$ can meet both $A$ and $A^{*}, \pi(A) \cap \pi\left(A^{*}\right)=\varnothing(4.4 \mathrm{~A})$. Thus $\pi(A)$ and $\pi\left(A^{*}\right)$ form an attractor-repeller pair for $\bar{f}$ and $\pi(\tilde{\alpha}(A, f))=\tilde{\alpha}(\pi(A), \bar{f})$ (4.3D). In particular, $\quad \pi(\operatorname{Comp} R(f))=\pi(\bigcup\{\tilde{\alpha}(A, f) \backslash A \mid A$ is an attractor of $f\})=$ $\bigcup\{\tilde{\alpha}(-(A), \bar{f}) \backslash \pi(A)\} \subset \operatorname{Comp} R(\bar{f})$. Also by $4.3 \mathrm{E} \quad \pi(R(f)) \subset R(\bar{f})$; thus $\pi(R(f))=R(\bar{f})$.

From $4.4 \mathrm{C}$ (the lemma) $\pi(R(f))$ is precisely the rest point set of $\bar{f}$; since this set is totally disconnected by $4.4 \mathrm{~B}, \bar{f}$ is a gradient flow. Also $4.4 \mathrm{C}$ implies there is a one-one correspondence between $R(\bar{f})$ and the components of $R(f)$.

If $\bar{f}$ is another gradient flow and $f>\bar{f}(h)$, then each component of $R(f)$ must be mapped to a single point of $R(\overline{\bar{f}})$ (by $4.3 \mathrm{E}$ and the fact that $R(\bar{f})$ consists of rest points). Thus a map $\bar{h}: \bar{X} \rightarrow \overline{\bar{X}}$ can be defined by $\bar{h}(\bar{x})=h\left(\pi^{-1}(\bar{x})\right)$. It is then easily seen that $\bar{h}$ is continuous and that $\bar{f}>\bar{f}(\bar{h})$.

THEOREM 4.4E. If $f$ and $f^{\prime}$ are $\omega$-similar, then so are their gradient parts.

Proof. Suppose $f>f^{\prime}(h)$ and $f^{\prime}>f\left(h^{\prime}\right)$ and let $\bar{f}$ and $\bar{f}^{\prime}$ denote the gradient parts with domination maps $\pi$ and $\pi^{\prime}$. Then since $f>\bar{f}^{\prime}\left(\pi^{\prime} \circ h\right), \bar{f}>\bar{f}^{\prime}$. Similarly $\bar{f}^{\prime}>\bar{f}$.

\section{Morse decompositions and filtrations}

Definition 5.1. If $s$ is the intersection of a quasi-attractor and a quasi-repeller, then $s$ will be called a quasi-Morse set.

If $s$ is the intersection of an attractor and a repeller, $s$ will be called a Morse set.

Note. A quasi-Morse set is the intersection of Morse sets.

LEMMA 5.2. $s$ is a quasi-Morse set if and only if $s=\Omega(s, f) \cap \Omega\left(s, f^{*}\right)$.

Proof. For any closed set $C, \Omega(C, f)$ is a quasi-attractor and $\Omega\left(C, f^{*}\right)$ a quasi-repeller; thus $\Omega(C, f) \cap \Omega\left(C, f^{*}\right)$ is a Morse set.

Suppose $s=A \cap \hat{A}^{*}$, where $A$ is a quasi-attractor and $\hat{A}^{*}$ is a quasi-repeller. Since $s$ is invariant, $\omega(s, f)=s$ so that $s \subset \Omega(s, f) \subset A$ and $s \subset \Omega\left(s, f^{*}\right) \subset \hat{A}^{*}$. Thus $s=\Omega(s, f) \cap \Omega\left(s, f^{*}\right)$.

LEMMA 5.3. If $s$ and $\bar{s}$ are quasi-Morse sets and $s \cap R(f)=\bar{s} \cap R(f)$, then $s=\bar{s}$.

Proof. First observe that for any flow $f$ on a compact metric space $Y, \Omega(R(f), f)=Y$. Namely $\Omega(R(f), f)$ is a quasi-attractor; if the complement in $Y$ were non-empty, it would contain a closed invariant set and any such meets $R(f)$ non-trivially.

Now if $x \in R(f \mid \bar{s})$, then $x$ is in $R(f)$ so in $\bar{s} \cap R(f)$ and so in $s$. Thus $\Omega(s, f) \supset$ $\Omega(R(f \mid \bar{s}), f) \supset \Omega(R(f \mid \bar{s}), f \mid \bar{s})=\bar{s}$. Similarly $\Omega\left(s, f^{*}\right) \supset \bar{s}$ and then $s \supset \bar{s}$. With the roles of $s$ and $\bar{s}$ interchanged, $\bar{s} \supset s$ and the result follows.

LEMMA 5.4. $s$ is a Morse set if and only if $s=\Omega(s, f) \cap \Omega\left(s, f^{*}\right)$ and $s \cap R(f)$ is closed and open in $R(f)$.

Proof. Suppose $s=A \cap \hat{A}^{*}$, where $A$ is an attractor and $\hat{A}^{*}$ is a repeller. By 5.3 $s=\Omega(s, f) \cap \Omega\left(s, f^{*}\right)$. Also $A \cap \hat{A}^{*} \cap R(f)=\tilde{\alpha}(A, f) \cap \tilde{\alpha}\left(\hat{A}^{*}, f^{*}\right) \cap R(f)$ since 
$\tilde{\alpha}(A, f) \backslash A$ and $\tilde{\alpha}\left(\hat{A}^{*}, f^{*}\right) \backslash \hat{A}^{*}$ are disjoint from $R(f)$. Thus $s \cap R(f)$ is closed and open in $R(f)$.

Suppose then that $s \cap R(f)$ is closed and open in $R(f)$ and that $s=\Omega(s, f) \cap$ $\Omega\left(s, f^{*}\right)$. Let $u$ be an open set containing $s$ with $u \cap R(f)=s \cap R(f)$. Since Comp $(u) \cap \Omega\left(s, f^{*}\right) \subset \operatorname{Comp} \Omega(s, f)$, for $x$ in the first set there exists an attractor $A(x)$ containing $\Omega(s, f)$ but not $x$. Since $\operatorname{Comp}(u) \cap \Omega\left(s, f^{*}\right)$ is compact, there is a finite collection $A_{1}, \ldots, A_{n}$ of attractors containing $\Omega(s, f)$ whose complements cover Comp $(u) \cap \Omega\left(s, f^{*}\right)$. Then $A \equiv A_{1} \cap \cdots \cap A_{n}$ is an attractor containing $\Omega(s, f)$ and disjoint from Comp $(u) \cap \Omega\left(s, f^{*}\right)$.

Similarly there can be found a repeller $\hat{A}^{*}$ containing $\Omega\left(s, f^{*}\right)$ which is disjoint from Comp $(u) \cap A$. Then $A \cap \hat{A}^{*}=\bar{s}$ is a Morse set contained in $u$ and containing s. Also $s \cap R(f)=u \cap R(f) \supset \bar{s} \cap R(f) \supset s \cap R(f)$ so $s \cap R(f)=\bar{s} \cap R(f)$. By 5.3 $s=\bar{s}$ and the result follows.

Definition 5.5. A Morse decomposition of $X$ with respect to $f$ means a (finite) ordered collection of Morse sets $\left\{s_{1}, \ldots, s_{n}\right\}$ such that

(a) $R(f)=\bigcup_{1}^{n} R(f) \cap s_{i}$

(b) for $i<j, \Omega\left(s_{i}, f\right) \cap s_{j}=\varnothing$ and $\Omega\left(s_{j}, f^{*}\right) \cap s_{i}=\varnothing$.

Definition. A filtration of $X$ with respect to $f$ is a finite increasing sequence $\phi=A_{0}, \ldots, A_{n}=X$ of attractors of $f$.

THEOREM 5.6. There is a one-one correspondence between filtrations and Morse decompositions such that to the filtration $\left\{\phi=A_{0}, \ldots, A_{n}=X\right\}$ there corresponds the Morse decomposition $s_{1}, \ldots, s_{n}$ with $s_{i}=A_{i} \cap A_{i-1}^{*}, i=1, \ldots, n$.

Then to the Morse decomposition $\left\{s_{1}, \ldots, s_{n}\right\}$ there corresponds the filtration $A_{i}=\Omega\left(s_{1} \cup \cdots \cup s_{i}, f\right), i=1, \ldots, n$, and $A_{0}=\phi$.

Proof. Let $\left\{\phi=A_{0}, \ldots, A_{n}=X\right\}$ be a filtration. The sets $s_{i} \equiv A_{i} \cap A_{i-1}^{*}$ are obviously disjoint Morse sets and satisfy $\Omega\left(s_{i}, f\right) \cap s_{j}=\Omega\left(s_{j}, f^{*}\right) \cap s_{i}=\phi$ whenever $i<j$. Suppose $x \in R(f)$. Then for some $i, x \in A_{i}$ and $x \notin A_{i-1}$. Since $x \notin A_{i-1}$ and $x \in R(f)$, $x \notin \tilde{\alpha}\left(A_{i-1}, f\right)$. Thus $x \in A_{i-1}^{*}$, hence $s_{i}=A_{i} \cap A_{i-1}^{*}$. Thus $\bigcup s_{i} \cap R(f)=R(f)$ and $\left\{s_{i}, \ldots, s_{n}\right\}$ is a Morse decomposition.

Now let $\left\{s_{1}, \ldots, s_{n}\right\}$ be a Morse decomposition and let $A_{i} \equiv \Omega\left(s_{1} \cup \cdots \cup s_{i}, f\right)$ for $i=1, \ldots, n$ and $A_{0}=\phi$. Then $A_{0}, \ldots, A_{n}$ is obviously an increasing sequence of quasi-attractors.

Suppose $j>i$. Then since $\Omega\left(s_{k}, f\right) \cap s_{j}=\phi$ for $k=1, \ldots, i$, it follows that $A_{i}=$ $\Omega\left(s_{1}, \ldots, s_{i}, f\right)=\bigcup_{k=1}^{i} \Omega\left(s_{k}, f\right)$ is disjoint from $s_{j}$. Thus $A_{i} \cap R(f)=\bigcup_{k=1}^{i} s_{k} \cap$ $R(f)$ and is a closed and open subset of $R(f)$. Since $A_{i}$ is a quasi-attractor, there must then be an attractor $\bar{A}_{i}$ containing $A_{i}$ such that $\bar{A}_{i} \cap R(f)=A_{i} \cap R(f) ; 5.3$ then implies $A_{i}=\bar{A}_{i}$ and is therefore an attractor. Thus $\left\{\phi=A_{0}, \ldots, A_{n}=X\right\}$ is a filtration.

Since $A_{i}$ contains $s_{1}, \ldots, s_{i}$ and is disjoint from $s_{i+1}, \ldots, s_{n}, A_{i} \cap A_{i-1}^{*}$ is a Morse set containing $s_{i}$ and disjoint from $s_{j}$ for $j \neq i$. This implies $A_{i} \cap A_{i-1}^{*} \cap R(f)=$ $s_{i} \cap R(f)$ and so by $5.3 s_{i}=A_{i} \cap A_{i-1}^{*}$ and the one-one correspondence is established. 


\section{REFERENCES}

[1] J. Auslander. Generalized recurrence in dynamical systems. Contr. Differential Equations 3 (1964), 65-74.

[2] R. Churchill. Isolated invariant sets in compact metric spaces. J. Differential Equations.

[3] C. Conley. On the continuation of invariant sets of a flow. Proceedings of the International Congress of Mathematicians 1970. Gauthier-Villars, Paris (1971), 909-913.

[4] C. Conley. On a generalization of the Morse index. Proceedings of the NRC-MRC Conference on Ordinary Differential Equations, Washington, DC, June 1971. Academic Press, New York (1972), 27-33.

[5] C. Conley. Some abstract properties of the set of invariant sets of a flow. Illinois J. Math. 16 (1972), 663-668.

[6] C. Conley \& R. Easton. Isolated invariant sets and isolating blocks. Trans. Amer. Math. Soc. 158 (1971), 35-61.

[7] J. Montgomery. Some perturbation theorems for flows on a compact metric space. Thesis. University of Wisconsin (1971). 